\title{
CRIMSONpublishers
}

http://www.crimsonpublishers.com

Case Report

Med Surg 0phthal Res

ISSN 2578-0360

\section{Ocular Histoplasmosis: About A Case}

\author{
Y Mouzari*, H Atidi, D Jâafari, M Chekhchar and M Kriet \\ Service of ophthalmology, Military Hospital of Marrakech Avicenne, Morocco
}

*Corresponding author: Y Mouzari, Service of ophthalmology, Military hospital of Marrakech Avicenne, Morocco

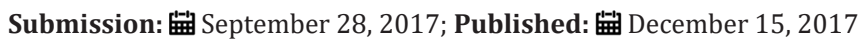

\begin{abstract}
Introduction: Histoplasmosis is a systemic mycosis present in an endemic state in many tropical or temperate regions. It is a pathology induced by Histoplasma capsulatum, dimorphic fungus thriving in wetlands and at moderate temperatures. In immunocompetent subjects, 95\% of clinical forms are asymptomatic. The preferred sites for this dissemination are pulmonary, medullary, hepatic, splenic and ganglionic. Exceptionally, the fungus can reach the eye.
\end{abstract}

Materials and methods: We report a case of ocular histoplasmosis in a 62-year-old patient.

Observation: It is a patient aged 62 years, diabetic under insulin for 5 months (discovery inaugural), amputated on the right side for a diabetic foot in October 2016, which showed a decrease in visual acuity appeared for 5 months. A complete ophthalmologic examination was carried out as part of the degenerative assessment of his diabetes, and he objectified: visual acuity at $1 / 10$ at right eye, $2 / 10$ at left, ocular tone at $16 \mathrm{Mmhg}$ at right eye and $14 \mathrm{mmhg}$ at left , biomicroscopic examination did'nt find tyndall in the anterior chamber, examination in the The bottom of eye showed: the presence of choroidal depigmented white at the retinal periphery with blamed haemorrhages, the absence of inflammation and inflammatory cells in the vitreous in both eyes, and the presence of peripapillary atrophy in the left eye.

Discussion: Ocular histoplasmosis syndrome (SS) is a deep mycosis with a pulmonary entry portal characterized by chorio retinal disorder caused by Histoplasma capsulatum, which is commonly found in endemic areas. The ocular damage remains quite exceptional since only 1.6 to $5.3 \%$ of patients with histoplasmosis are affected. The diagnosis of ocular histoplasmosis is made by clinical examination. The fundus of the eye can find histo spots, peripapillary atrophy, macular disciform lesions active or not and never finds inflammatory cells in the vitreous and the anterior segment. The systemic antifungal treatments used in the treatment of histoplasmosis have little use in the treatment of ophthalmologic involvement because the fungus does not appear to be in the eye at the time of ophthalmologic diagnosis. The treatment of eye damage is therefore very specific.

Conclusion: Ocular histoplasmosis is a fungal infection that has long been underestimated because of the asymptomatic or benign character of the primary infection. Indeed, this ophthalmological pathology, of random evolution and with heavy clinical consequences, is very little known and rarely sought, whereas an early diagnosis improves its management and hence its functional prognosis.

Keywords: Ocular; Histoplasmosis; Histo spots; Peripapillary atrophy; Neovascularization; Systemic antifungal treatments

\section{Introduction}

Histoplasmosis is a systemic mycosis present in an endemic state in many tropical or temperate regions. It is a pathology induced by Histoplasma capsulatum, dimorphic fungus thriving in wetlands and at moderate temperatures. In immunocompetent subjects, $95 \%$ of clinical forms are asymptomatic. The preferred sites for this dissemination are pulmonary, medullary, hepatic, splenic and ganglionic. Exceptionally, the fungus can reach the eye.

Ocular abnormalities associated with acute disseminated histoplasmosis were thus described for the first time in 1942.

Histoplasma capsulatum can cause three types of ophthalmological pathologies in humans: histoplasmic endophthalmitis, solitary choroidal histoplasmic granuloma, and presumed ocular histoplasmosis syndrome (POBS).

\section{Observation}

It is a patient aged 62 years, diabetic under insulin for 5 months (discovery inaugural), amputated on the right side for a diabetic foot in October 2016, which showed a decrease in visual acuity appeared for 5 months .

A complete ophthalmologic examination was carried out as part of the degenerative assessment of his diabetes and was objectified: visual acuity $1 / 10$ th in the right eye and 2/10th in the left eye, ocular tone At $16 \mathrm{mmh}$ at the right eye and at $14 \mathrm{mmhg}$ at the left eye, biomicroscopic examination did not find any tyndall in the anterior chamber, examination in the fundus showed: the presence of tasks white depigmented choroidal at the retinal periphery with splinter haemorrhages at the posterior pole and the absence of inflammation and inflammatory cells in the vitreous at the two eyes, and the presence of a peripapillary atrophy at the left eye. 
A fluorescein angiography objectified: a diffusion image related to retinal neovascularization and under a window effect at the histospots (Figure 1).
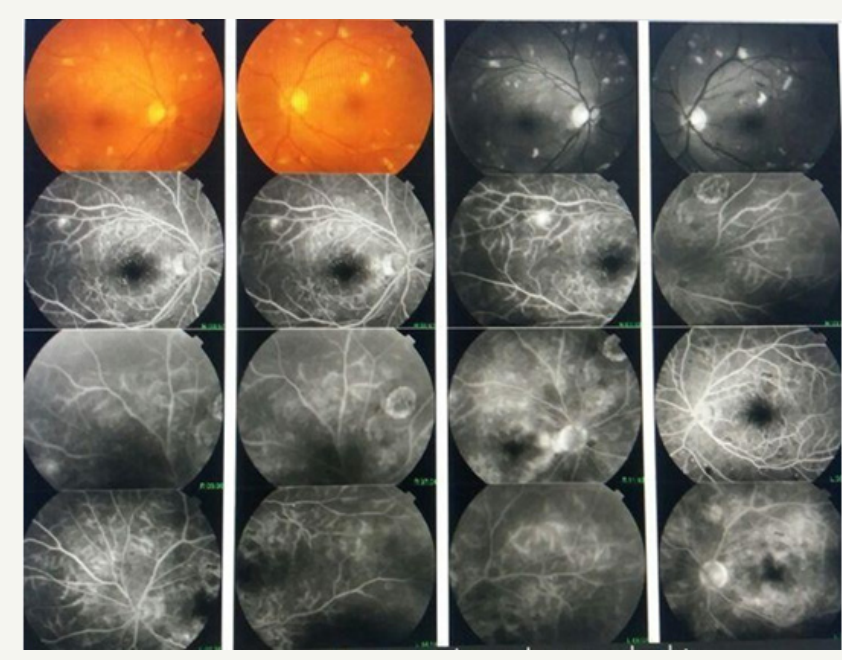

Figure 1: fluorescein.

Retinography-Retinal angiography with

\section{Discussion}

Ocular histoplasmosis syndrome (OHS) is a deep mycosis with a pulmonary entry pathway characterized by chorio retinal disorder caused by Histoplasma capsulatum, which is commonly found in endemic areas. The ocular damage remains quite exceptional since only 1.6 to $5.3 \%$ of patients with histoplasmosis are affected. Histoplasma capsulatum can cause three types of ophthalmologic pathologies in humans: histoplasmic endophthalmitis, rare choroidal histoplasmic granuloma and the presumed ocular histoplasmosis syndrome (POBS).

PORS is a cause of vision loss. Its classical description is the triad [3]: discrete choroid atrophic scars called histo spots, peripapillary atrophy, sub retinal neovascularizations, triad to which must be added the absence of inflammation in the window. His diagnosis is based on a set of clinical arguments. Although the lesions are not symmetrical in the initial presentation, lesions are very common in both eyes.

Table 1: Differential Diagnosis of Ocular Histoplasmos.

\begin{tabular}{|c|}
\hline Diagnostics Differentiels de I'histoplasmose Oculaire \\
\hline SarcoYdose, Tuberculose, Coccidiomycose, Cryptococcose \\
\hline Choroklite multifocale, Panuvtite \\
Toxoplasmose \\
\hline Chorioretinites de Birdshot \\
\hline ChoroIdopathie interne ponctuee \\
\hline
\end{tabular}

The diagnosis of ocular histoplasmosis is made by clinical examination. The fundus of the eye can find histo spots, peripapillary atrophy, macular disciform lesions active or not and never finds inflammatory cells in the vitreous and the anterior segment. We currently do not have antigen detection methods, and we are limited by insufficiently sensitive and specific serological methods to form a powerful diagnostic tool in histoplasmosis [3] (Table 1).

The course of the pathology is made up of exacerbations and recurrences occurring mostly on histo stain scars already present. The severity of clinical manifestations related to POBS depends on the location of the histo spots and their presence or not on the macula. Studies have shown that in the case of asymptomatic macular lesions on one eye, there is a $20-25 \%$ risk of developing macular lesions in the contralateral eye during recurrences $[4,5]$. They also showed that $58 \%$ of the eyes with untreated macular choroidal neovascular showed a marked decrease in visual acuity. Another study showed that $16.6 \%$ of the eyes developed new choroidal and retinal infiltrates in areas that were clinically and angiographically normal ten years earlier [6]. Ocular histoplasmosis is therefore a chronic pathology, of random evolution and with heavy clinical consequences.

The systemic antifungal treatments used in the treatment of histoplasmosis have little use in the treatment of ophthalmologic involvement because the fungus does not appear to be in the eye at the time of ophthalmologic diagnosis. The treatment of eye damage is therefore very specific. Therapeutic abstention is proposed for patients with only histo spots, however, regular monitoring by an ophthalmologist is necessary because of the risk of macular involvement. In case of scars at the level of the macular area, a selfmonitoring by Amsler grid is desirable. For corticotherapy, it seems to have a beneficial effect on the neovascularizations associated with this syndrome and, although their utility has been studied little, they are used by many clinicians.

A study of a series of 18 patients showed that oral corticosteroid therapy with prednisone made it possible in the short term to improve the visual acuity of patients and in the long term to stabilize retro-foveal CNVs. It also appears to be of particular interest in the prevention of recurrences after retrovoveal surgery and in patients unable to benefit from surgery [7].

However, since argon laser photocoagulation has shown a definite benefit in the treatment of CNVs outside the avascular foveal zone, the use of corticosteroids should be reserved for situations in which NVCs are located at the level of the fovea [8]. This corticosteroid therapy may be necessary from 2 weeks to 2 years depending on the severity of the attack. As for laser photocoagulation, numerous clinical studies have shown that it can reduce the risk of severe loss of secondary vision to $\mathrm{CNV}$ in patients with PORS. An American study recently concluded that laser treatment is a very cost-effective treatment both from a monetary point of view and in terms of improving quality of life [9].

\section{Conclusion}

Ocular histoplasmosis is a fungal infection which has long been underestimated because of the asymptomatic or benign nature of the 
primary infection. It would be desirable for an information work to be carried out among practitioners so that, Onset of choroidopathy in the course of histoplasmosis, an ophthalmologic examination is performed in any patient presenting with disseminated histoplasmosis. Indeed, this ophthalmological pathology, of random evolution and with heavy clinical consequences, is little known and very rarely sought, whereas an early diagnosis improves its management, and therefore, its functional prognosis.

\section{References}

1. Mina Laghmari (2014) sd d'histoplasmose oculaire presumé; Omar Lezrek 'Pan Afr Med J 18: 268.

2. El Mellaoui M, El Ouafi A, El Hansali Z, Bouzidi A, Iferkhas, et al. (2015) Presumed ocular histoplasmosis syndrome. J Fr Ophtalmol 38(9): 892893.

3. Ciulla TA, Piper He, Xiao M, Wheat LJ (2001) Presumed ocular histoplasmosis syndrome: update on epidemiology, pathogenesis, and photodynamic, antiangiogenic, and surgical therapies. CUIT Opin Ophthalmol 12(6): 442-449.
4. Lewis ML, Van Newkirk M, Gass JD (1980) Follow-up study ofpresumed ocular histoplasmosis syndrome. Ophthalmology 87(5): 390-399.

5. Sawelson H, Goldberg RE, Annesley WH (1976) Presumed ocular histoplasmosissyndrome: The fellow eye. Arch Ophthalmol 94(2): 221224

6. Watzke RC, Claussen R (1981) The long-term course of multifocal choroiditis (presumed ocular histoplasmosis). Am J Ophthalmol 91(6): 750-760.

7. Martidis A, Miller DG, Cuilla TA, Danis RP, Moorthy RS (1999) Corticosteroids as an antiangiogenic agent for histoplasmosis-re1ated subfoveal choroidal neovascularization. Journal of Ocular Pharmacology \& Therapeutics 15(5): 425-428.

8. Gass JDM (1987) Stereoscopie atlas of macular diseases Diagnosis and treatment. StLouis Mosby 230. Macular Photocoagulation S 60(6): 604605.

9. Brown GC, Brown MM, Sharma S, Busbee B, Brown H (2000) Incremental cost effectiveness of laser therapy for choroidal neovascularization associed with histoplasmosis. Retina 20(4): 331-337. 